\title{
The second release of the Torun catalogue of Galactic post-AGB objects: New classification scheme
}

\author{
Ryszard Szczerba ${ }^{1}$, Natasza Siódmiak ${ }^{1}$, Grazyna Stasińska ${ }^{2}$, Jozef \\ Borkowski $^{1}$, Pedro García-Lario ${ }^{3}$, Olga Suárez ${ }^{4,5}$, Marcin Hajduk ${ }^{1}$, \\ and D. Anibal García-Hernández ${ }^{6,7}$ \\ ${ }^{1}$ N. Copernicus Astronomical Center, Torun, Poland \\ ${ }^{2}$ LUTH, Observatoire de Paris, CNRS, France \\ ${ }^{3}$ Herschel Science Centre, European Space Astronomy Centre, ESA, Spain \\ ${ }^{4}$ Universite de Nice Sophia Antipolis, CNRS, France \\ ${ }^{5}$ Laboratorio de Astrofísica Espacial y Física Fundamental, INTA, Spain \\ ${ }^{6}$ Instituto de Astrofísica de Canarias, Spain \\ ${ }^{7}$ Universidad de La Laguna, Spain
}

\begin{abstract}
The investigation of post-AGB objects (proto-planetary nebulae) is very important from the standpoint of physical and chemical changes occurring during the late stages of stellar evolution. The Torun catalogue of Galactic post-AGB and related objects is an evolutive catalogue containing astrometric, photometric and spectroscopic data as well as HST images for all known post-AGB objects and candidates in our Galaxy. This free-access catalogue can serve as an ideal tool to study different groups of post-AGB objects, especially due to the fact that all information is gathered in one place. The second release of our catalogue introduces a simple classification scheme of post-AGB objects and includes a significant number of new objects, photometric data, spectra and images. Here, using objects from the catalogue we consider the problem of the termination of the AGB phase.
\end{abstract}

Keywords. catalogs, stars: AGB and post-AGB

\section{The catalogue}

The post-AGB objects gathered in our catalogue are stars that left the AGB but are still not hot enough to ionize the surrounding matter. This definition does not imply that the objects will become planetary nebulae $(\mathrm{PN})$, since stellar ejecta prior to this stage may have dispersed in the interstellar medium well before the remnant star is hot enough to ionize them. The second release of the catalogue (http://www.ncac.torun.pl/postagb2 Szczerba et al. 2011, A\&A in revision) gives on-line access to about 480 likely and possible post-AGB objects, including about 110 RV Tau stars \& 70 helium stars. There are also about 70 unlikely post-AGB objects, which sometimes are counted in the literature as proto-PNe or post-AGBs.

The main tool to classify a post-AGB candidate is its position in the IRAS color-color diagram (CCD) and its IRAS variability, supplemented by some additional criteria, if necessary. We count an object as a likely post-AGB if it lies below the dotted line in the CCD (Fig. 1), has IRAS variability index smaller than 40 and is not a PN (CCDpos). For objects located above the line we used additional criteria, like: double peaked 1612 $\mathrm{MHz} \mathrm{OH}$ maser emission, luminosity class I, etc (CCDoth). A detailed description of the adopted criteria can be found in Szczerba et al. (2011). We have also distinguished 


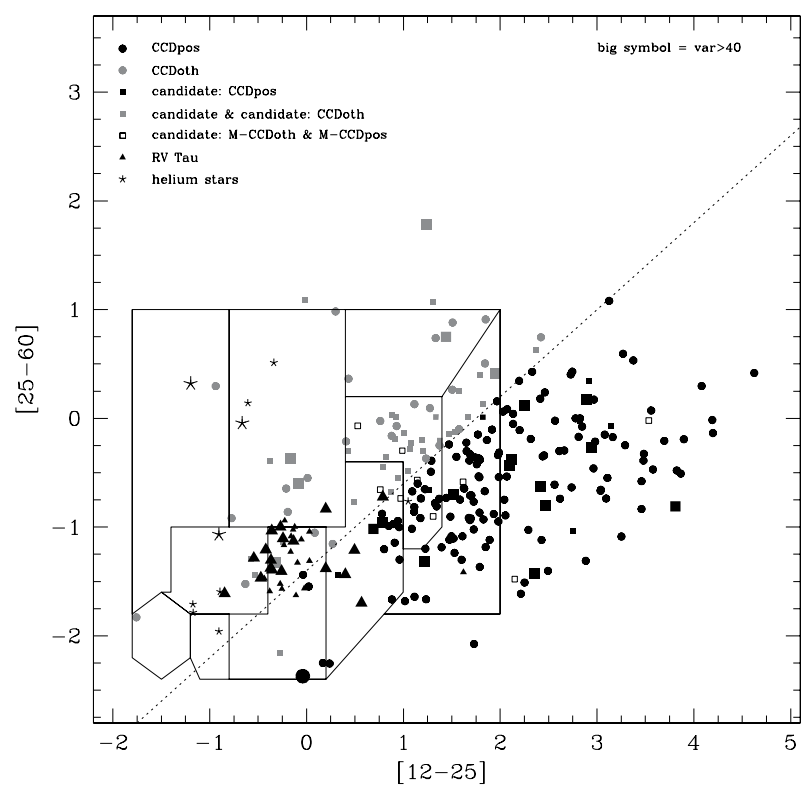

Figure 1. IRAS color-color diagram for objects from the Torun catalogue with IRAS fluxes.

a group of M-type post-AGB(?) stars (M-CCDpos; M-CCDoth), which are discussed in Sect. 2 .

The second release of the catalogue is enriched in: a) photometry from GSC 2.3, b) optical spectra from Suárez et al. (2006) and Pereira \& Miranda (2007) for 120 sources, c) HST images for $\sim 100$ sources, d) classification of spectral energy distribution (SED) according to the van der Veen et al. (1989) scheme (additionally, we introduced class 0 for objects with almost no IR excess), e) $V$ and $R$ long-term photometric variability from Hrivnak et al. (2010) for 12 sources, f) IRAS variability index, g) dominant chemistry photospheric and/or circumstellar.

\section{M-type objects and the end of AGB}

There are several objects in our catalogue with double-peaked SEDs and spectral type $\mathrm{M}$ implying an effective temperature around $3000 \mathrm{~K}$ and yet called in the literature post-AGB objects. While double-peaked SED suggests the end of AGB phase, the Mtype contradicts this conclusion. However, the only M-type object (IRAS 18420-0512) resolved by HST shows circumstellar arcs (Sahai et al. (2007)). Therefore, we postulate that M-type objects are at the end of their "puffing" phase during AGB and are just entering the post-AGB stage of stellar evolution, so they could be called the "transition post-AGB objects".

\section{References}

Hrivnak, B. J., Lu, W., Maupin, R. E., \& Spitzbart, B. D. 2010, ApJ, 709, 1042

Pereira, C. B. \& Miranda, L. F. 2007, A\&A, 462, 231

Sahai, R., Morris, M., Sánchez Contreras, C., \& Claussen, M. 2007, AJ, 134, 2200

Suárez, O., García-Lario, P., Manchado, A., Manteiga, M., Ulla, A., \& Pottasch, S. R. 2006, $A \& A, 458,173$

van der Veen, W. E. C. J., Habing, H. J., \& Geballe, T. R. 1989, A\& A, 226, 108 